\title{
Ballistics Model for Particles on a Horizontal Plane in a Vacuum Propelled by a Vertically Impinging Gas Jet
}

\author{
J. E. LANE ${ }^{1}$ AND P. T. METZGER ${ }^{2}$ \\ ${ }^{1}$ ASRC Aerospace, Space Life Sciences Lab, Kennedy Space Center, Florida, \\ USA \\ ${ }^{2}$ Granular Mechanics and Regolith Operations Lab, NASA, Kennedy Space \\ Center, Florida, USA
}

A simple trajectory model has been developed and is presented. The particle trajectory path is estimated by computing the vertical position as a function of the horizontal position using a constant horizontal velocity and a vertical acceleration approximated as a power law. The vertical particle position is then found by solving the differential equation of motion using a double integral of vertical acceleration divided by the square of the horizontal velocity, integrated over the horizontal position. The input parameters are: $x_{0}$ and $y_{0}$, the initial particle starting point; the derivative of the trajectory at $x_{0}$ and $y_{0}, s_{0}=s\left(x_{0}\right)=d x(y) /\left.d y\right|_{y=y_{0}}$; and $b$ where $b x_{0} / y_{0}$ is the final trajectory angle before gravity pulls the particle down. The final parameter $v_{0}$ is an approximation to a constant horizontal velocity. This model is time independent, providing vertical position $x$ as a function of horizontal distance $y$ :

$$
x(y)=\left(x_{0}+s_{0}\left(y-y_{0}\right)\right)+\left(b x_{0}-s_{0} y_{0}\right)\left(\frac{y-y_{0}}{y_{0}}-\ln \frac{y}{y_{0}}\right)-\frac{g\left(y-y_{0}\right)^{2}}{2 v_{0}^{2}}
$$

The first term on the right in the above equation is due to simple ballistics and a spherically expanding gas so that the trajectory is a straight line intersecting $(0,0)$, which is the point at the center of the gas impingement on the surface. The second term on the right is due to vertical acceleration, which may be positive or negative. The last term on the right is the gravity term, which for a particle with velocities less than escape velocity will eventually bring the particle back to the ground. The parameters $b, s_{0}$, and in some cases $v_{0}$, are taken from an interpolation of similar parameters determined from a CFD simulation matrix, coupled with complete particle trajectory simulations.

Keywords lunar surface, regolith, ballistics, particle trajectory, equation of motion, rarefied gas jet

\section{Introduction}

In preparation for the Apollo program, Leonard Roberts of the NASA Langley Research Center developed a remarkable analytical theory that predicts the blowing of lunar soil and dust beneath a rocket exhaust plume. Roberts assumed that the erosion rate was determined by the excess shear stress in the gas (the amount of shear stress greater than what causes grains to roll). The acceleration of particles to their final velocity in the gas consumes a portion of the shear stress. The erosion rate continues to increase until the excess shear stress is exactly consumed, thus determining the erosion rate. Roberts calculated the largest and smallest particles that could be eroded based on forces at the particle scale, but the erosion rate equation assumed that only one particle size existed in the soil. He assumed that particle ejection angles were determined entirely by the shape of the terrain, which acts like a ballistic ramp, with the particle aerodynamics being negligible. The predicted erosion rate and the upper limit of particle size appeared to be within an order of magnitude of small-scale terrestrial experiments but could not be tested more quantitatively at the time. The lower limit of particle size and the predictions of ejection angle were not tested.

We observed in the Apollo landing videos that the ejection angles of particles streaming out from individual craters were time-varying and correlated to the Lunar Module thrust, thus implying that particle aerodynamics dominate. We modified Roberts' theory in two ways. First, we used ad hoc the 
ejection angles measured in the Apollo landing videos, in lieu of developing a more sophisticated method. Second, we integrated Roberts' equations over the lunar-particle size distribution and obtained a compact expression that could be implemented in a numerical code. We also added a material damage model that predicts the number and size of divots which the impinging particles will cause in hardware surrounding the landing rocket. Then, we performed a long-range ballistic analysis for the ejected particulates.

An ongoing activity since that time has been the development of particle trajectory simulations in order to improve estimates of trajectory angle and speed as a function of particle size and initial starting point on the ground relative to the engine nozzle. Four distinct models have been used to attack this problem:

1. Computational Fluid Dynamics (CFD) code (Fluent ${ }^{\mathrm{TM}}$ ) - the simulation is set up to characterize the gas flow from a rocket engine impinging vertically on a rigid horizontal surface. The surface may have other specific features that replicate shallow circular craters or vertical berms to stop the high velocity spray of regolith from the surface.

2. Particle Trajectory with Quadratic Shepard's Interpolation (PTQ) - a stepped integration method to solve a single particle's differential equations of motion. The CFD computed gas properties, temperature, density, and vector velocity, are the input to this model, where the gas provides the force to propel a single particle of diameter $D$ from the ground at an initial starting point, $\mathbf{r}_{0}$.

3. Empirical Trajectory Path (ETP) model - an empirical time independent function is used to estimate a 2D trajectory path of a single particle, including path shape and angle. The horizontal velocity $v_{\mathrm{y}}$, initial starting point $\left(x_{0}, y_{0}\right)$, and two shape parameters $b$ and $c$ constitute the model's input parameters.. The shape parameters are determined by fitting the empirical function to associated PTQ trajectories from step 2. A matrix of ETP shape parameters and horizontal velocities are generated from a matrix of particle variables, such as of particle size, starting locations, engine height, and engine thrust, and by fitting the ETP model to the PTQ generated trajectories. Using a simple $N$-dimensional interpolation algorithm, ETP model parameters can then be estimated for any particle size, starting point, as well as engine height above the ground.

4. Physical Trajectory Path (PTP) model - The same overall procedure to obtain parameter interpolations used in step 3 is used in this case, but the empirical function is replaced with a special solution to the differential equations of motion. This solution is based on an estimate of vertical particle acceleration and particle horizontal velocity. This model uses two shape factors, $s_{0}$ and $b$, as well as horizontal velocity $v_{0}$ and initial starting point $\left(x_{0}, y_{0}\right)$. The only difference in the parameter definitions between ETP and PTP is the parameter $c$ vesus $s_{0}$.

\section{Empirical Model}

In previous work (Lane, 2010), an empirical trajectory path model was investigated to describe the trajectory path of particles on the Lunar surface under a rocket plume:

$$
x(y)=b x_{0}\left(\left(\frac{y}{y_{0}}\right)^{c}+\frac{1}{b^{c}}-1\right)^{1 / c}-\frac{g}{2}\left(y-y_{0}\right)^{2} v_{y}^{-2},
$$

with a gravity free $(g=0)$ trajectory angle:

$$
\theta=\tan ^{-1}\left(b \frac{x_{0}}{y_{0}}\right)
$$


The model of Equation (1) mimics the general trajectory path behavior of particles on a smooth horizontal surface propelled by a vertically impinging rarefied gas jet. Figure 1 shows a plot of Equation (1) where $x$ is the vertical direction (direction of gravity), $y$ is the horizontal direction, and $\left(x_{0}, y_{0}\right)$ is the initial starting point of the particle. When $b=1$, with gravity $g=0$, the resulting path is that of a straight line which intersects the points $(0,0)$ and $\left(x_{0}, y_{0}\right)$. The trajectory angle in this case is $\tan ^{-1}\left(x_{0} / y_{0}\right)$. In most cases the parameter $b \neq 0$ so that the final gravity free trajectory angle is $\tan ^{-1}\left(b x_{0} / y_{0}\right)$. The parameter $c$ in Equation (1) adjusts the curvature of $x(y)$.

The "gravity free" region in this context is determined by the last term on the right hand side of Equation (1). When this term is much smaller than the first term on the right hand side, the trajectory angle will be a constant value equal to $\tan ^{-1}\left(b x_{0} / y_{0}\right)$. The trajectory path model parameter $v_{y}$ of Equation (1) is the horizontal velocity of the particle. When this value is very large, from 100 to 2400 (escape velocity) $\mathrm{m} / \mathrm{s}$, the gravity term ( $g=1.622 \mathrm{~m} \mathrm{~s}^{-2}$ on the lunar surface) has minimal effect within a radius of 20 to $30 \mathrm{~m}$ for smaller particles less than $100 \mu \mathrm{m}$ in diameter. However, regardless of the values of $g$ or $v_{y}$ the straight trajectory path defined by $\tan ^{-1}\left(b x_{0} / y_{0}\right)$ will eventually lose out to the gravity term and the path will curve back to intersect the surface (unless the particle velocity exceeds escape velocity).

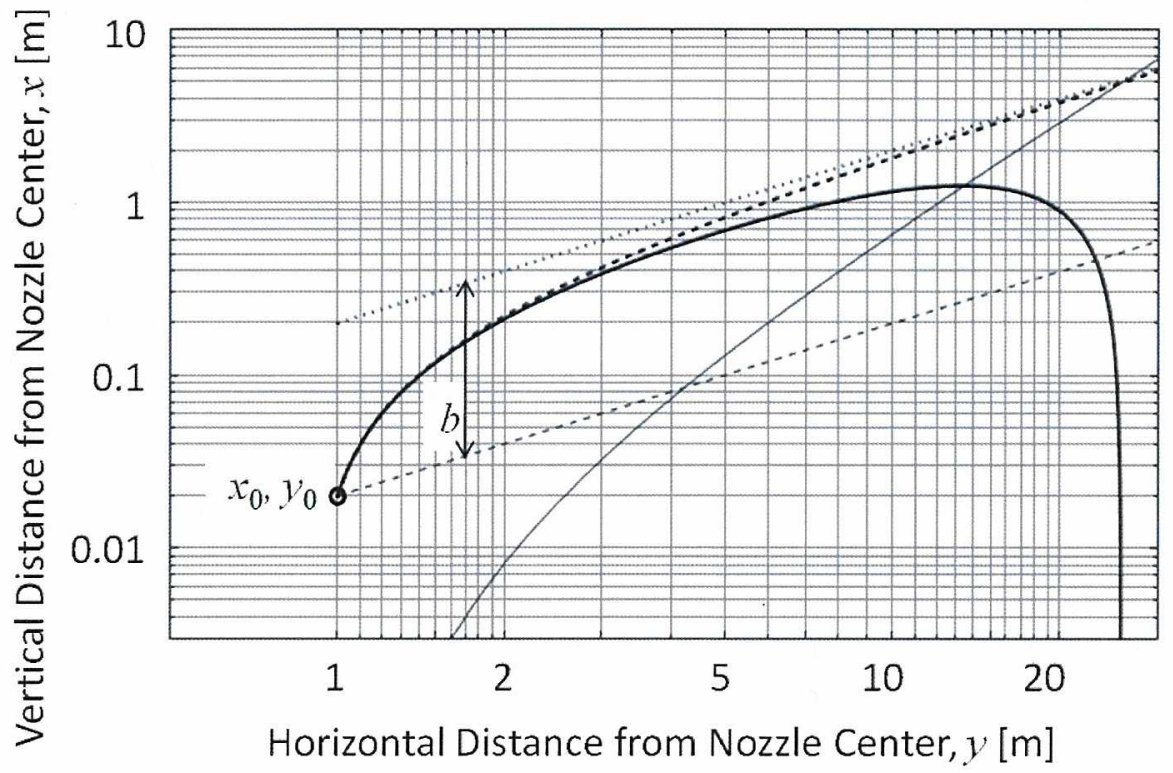

Figure 1. Solid thick line: example trajectory path from Equation (1) with $x_{0}=0.02[\mathrm{~m}], y_{0}=1.0[\mathrm{~m}], b=$ $10, c=1, v_{y}=10[\mathrm{~m} / \mathrm{s}]$; solid line: gravity term (last term on right hand side of Equation (1); dashed line: $\tan ^{-1}\left(x_{0} / y_{0}\right)$ slope line; dotted line: $\tan ^{-1}\left(b x_{0} / y_{0}\right)$ line; thick dashed line: gravity free version of Equation

(1), with $g=0$.

\section{CFD Trajectories Interpolation Matrix}

Table 1 shows a matrix of $N$ particle values (size and starting location), $\mathbf{P}_{i}=\left(D_{i}, x_{0 \mathrm{i}}, y_{0 \mathrm{i}}\right)^{\mathrm{T}}$, with the corresponding of fit of Equation (1) to the final CFD based trajectories. The fitted model parameters are $\mathbf{X}_{i}=\left(b_{i}, c_{\mathrm{i}}, v_{y \mathrm{i}}\right)^{\mathrm{T}}$ :

$$
\mathbf{P}=\left(\begin{array}{c}
D \\
x_{0} \\
y_{0}
\end{array}\right) \quad \mathbf{X}=\left(\begin{array}{c}
b \\
c \\
v_{y}
\end{array}\right) \quad \mathbf{P}_{i}=\left(\begin{array}{c}
D_{i} \\
x_{0_{i}} \\
y_{0 i}
\end{array}\right) \quad \mathbf{X}_{i}=\left(\begin{array}{c}
b_{i} \\
c_{i} \\
v_{y_{i}}
\end{array}\right)
$$


The values in Table 1 are found by first computing a matrix of full particle trajectories generated by postprocessing CFD output data using the equations of motion, Equation (1.0) and considering the details of particle drag and lift (Lane, 2010). Figure 2 shows trajectories generated by fitting the model of Equation (1) CFD based trajectories. The result of the fit for $N$ trajectories is shown in Table 1.

Table 1. Empirical model parameters found by fitting Equation (1) to a matrix of CFD trajectory solutions for a range of particle sizes $D$ and initial starting points $x_{0}, y_{0}$.

\begin{tabular}{|c|c|c|c|c|c|}
\hline$D$ & $x_{0}$ & $y_{0}$ & $b$ & $C$ & $v_{y}$ \\
\hline 1 & 0.01 & 0.88779 & 5.061 & 0.6445 & 1997 \\
\hline 1 & 0.01 & 1.08699 & 4.715 & 0.7719 & 1770 \\
\hline 1 & 0.01 & 1.33089 & -99 & -99 & -99 \\
\hline 1 & 0.01 & 1.62951 & -99 & -99 & -99 \\
\hline 1 & 0.01 & 1.99514 & 8.221 & 0.58 & 640.4 \\
\hline 1 & 0.01 & 2.4428 & -99 & -99 & -99 \\
\hline 1 & 0.01 & 2.99091 & -99 & -99 & -99 \\
\hline 1 & 0.01 & 3.662 & 24.78 & 0.7374 & 414.4 \\
\hline 1 & 0.01 & 4.48367 & 312.7 & 1.163 & 480.3 \\
\hline 1 & 0.01 & 5.4897 & -99 & -99 & -99 \\
\hline 1 & 0.01 & 6.72146 & 49.69 & 1.269 & 346.1 \\
\hline 1 & 0.1 & 0.88779 & 1.953 & 0.5507 & 2381 \\
\hline 1 & 0.1 & 1.08699 & -99 & -99 & -99 \\
\hline 1 & 0.1 & 1.33089 & 2.254 & 0.7018 & 2228 \\
\hline 1 & 0.1 & 1.62951 & -99 & -99 & -99 \\
\hline 1 & 0.1 & 1.99514 & 2.402 & 0.7626 & 1872 \\
\hline 1 & 0.1 & 2.4428 & -99 & -99 & -99 \\
\hline 1 & 0.1 & 2.99091 & 2.42 & 0.8279 & 1098 \\
\hline 1 & 0.1 & 3.662 & -99 & -99 & -99 \\
\hline 1 & 0.1 & 4.48367 & 3.123 & 0.7227 & 494.7 \\
\hline 1 & 0.1 & 5.4897 & -99 & -99 & -99 \\
\hline 1 & 0.1 & 6.72146 & 5.356 & 0.8368 & 311.8 \\
\hline 1000 & 0.01 & 0.88779 & 3.177 & 0.7301 & 84.87 \\
\hline 1000 & 0.01 & 1.08699 & -99 & -99 & -99 \\
\hline 1000 & 0.01 & 1.33089 & -99 & .99 & .99 \\
\hline 1000 & 0.01 & 1.62951 & 4.057 & 0.8624 & 41.21 \\
\hline 1000 & 0.01 & 1.99514 & 4.296 & 0.7009 & 26.22 \\
\hline 1000 & 0.01 & 2.4428 & 10.78 & 0.5 & 17.12 \\
\hline 1000 & 0.01 & 2.99091 & -99 & -99 & -99 \\
\hline 1000 & 0.01 & 3.662 & 1.739 & 2 & 4.296 \\
\hline 1000 & 0.01 & 4.48367 & -99 & -99 & -99 \\
\hline 1000 & 0.01 & 5.4897 & -99 & -99 & -99 \\
\hline 1000 & 0.01 & 6.72146 & 1 & 0.5 & 0 \\
\hline 1000 & 0.1 & 0.88779 & 1.591 & 0,4195 & 133.6 \\
\hline 1000 & 0.1 & 1.08699 & -99 & -99 & -99 \\
\hline 1000 & 0.1 & 1.33089 & 2.136 & 0.7379 & 116.3 \\
\hline 1000 & 0.1 & 1.62951 & -99 & -99 & -99 \\
\hline 1000 & 0.1 & 1.99514 & 2.289 & 0.8039 & 88.73 \\
\hline 1000 & 0.1 & 2.4428 & -99 & -99 & -99 \\
\hline 1000 & 0.1 & 2.99091 & 2.239 & 0.8885 & 43.09 \\
\hline
\end{tabular}



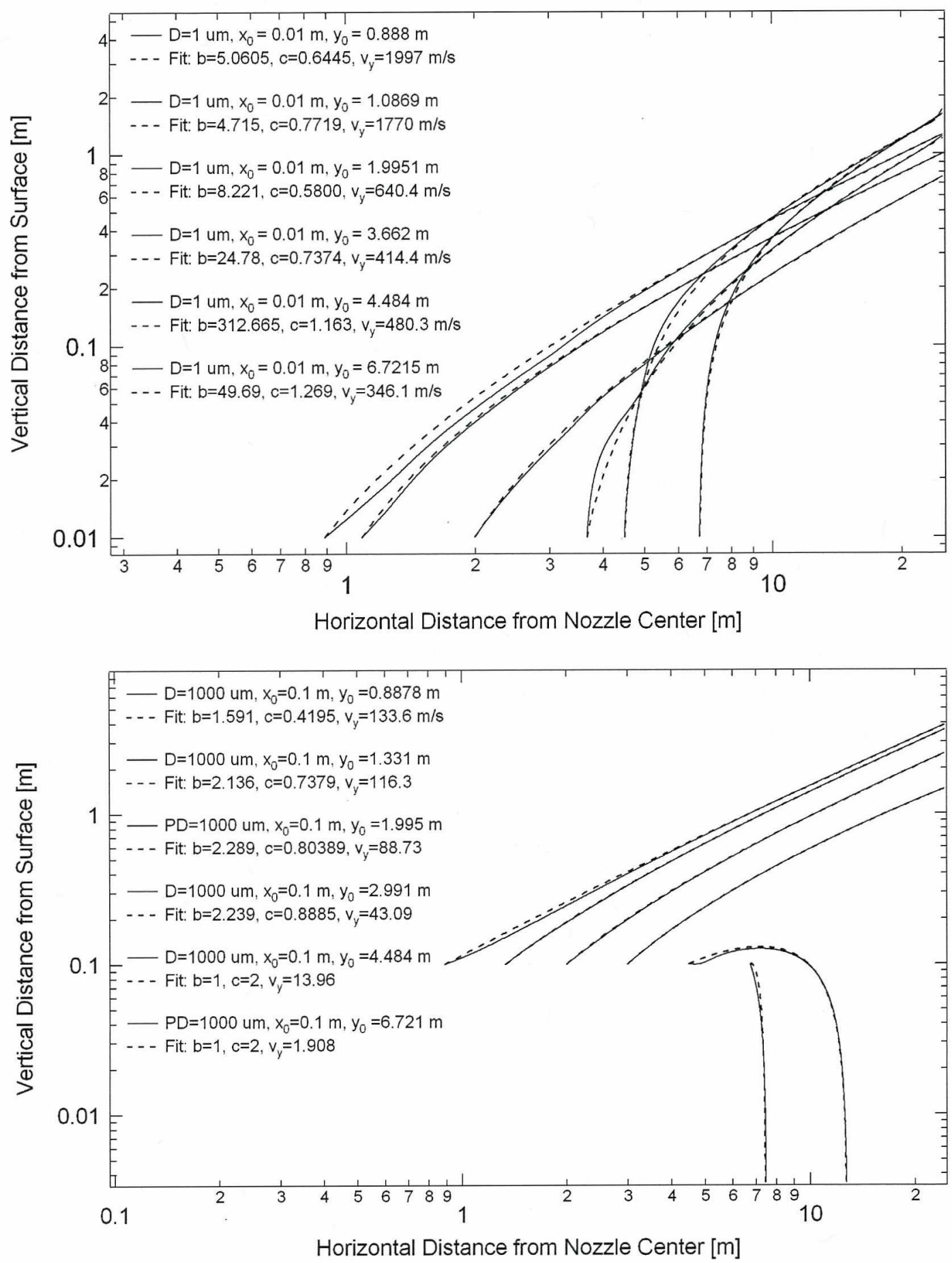

Figure 2. Solid lines: Full particle trajectories generated from post-processing of CFD simulations; dashed lines: trajectories generated by fitting the model of Equation (1) to solid lines. Top: $D=1 \mu \mathrm{m}$ and $x_{0}=$ $0.01 \mathrm{~m}$; bottom: $D=1000 \mu \mathrm{m}$ and $x_{0}=0.1 \mathrm{~m}$.

Note that an entry of "-99" in Table 1 corresponds to no fit data available. Each entry in Table 1 is the result of a full CFD based trajectory, but not all of those entries were fitted to Equation (1). The number of useable entries $N$ from Table 1 is therefore 21, in this example. 
Once a matrix of model parameter values has been determined, an estimated set of parameters $\mathbf{P}$ can be computed for any arbitrary set of input values $\mathbf{X}$ using an application of Shepard's interpolation algorithm (Shepard, 1968) to the matrix defined by Table 1:

$$
\mathbf{P}=\frac{\sum_{i=1}^{N} w_{i} \mathbf{P}_{i}}{\sum_{i=1}^{N} w_{i}},
$$

where,

$$
w_{i}=\frac{1}{\left(\left(\mathbf{X}-\mathbf{X}_{i}\right) \cdot\left(\mathbf{X}-\mathbf{X}_{i}\right)\right)^{\mu}},
$$

where $\mu=3$ seems to be a good choice for this application.

\section{Theoretical Model}

The general equation of motion of a particle of mass $m$ acted upon by a force $\mathbf{F}$ is:

$$
m \ddot{\mathbf{r}}=\mathbf{F} .
$$

Since we are interested in the trajectory path $P(y)$, the following substitution eliminates the time variable from the differential equation of Equation (6):

$$
\begin{aligned}
\ddot{\mathbf{r}} & =\frac{d}{d t} \frac{d}{d t} \mathbf{r} \\
& =\frac{d y}{d t} \frac{d}{d y} \cdot \frac{d y}{d t} \frac{d}{d y} \mathbf{r} \\
& =v_{y} \frac{d}{d y} \cdot v_{y} \frac{d}{d y} \mathbf{r} \\
& =v_{y}\left(\frac{d v_{y}}{d y} \cdot \frac{d \mathbf{r}}{d y}+v_{y} \frac{d^{2} \mathbf{r}}{d y^{2}}\right)
\end{aligned}
$$

Note that in Equation (7), the independent variable is $y$ while the dependent variable is $x=P(y)$. This seemingly backwards convention comes about because the axis of the gas jet is defined to be along the vertical $x$-axis while the ground plane lies along the $y$-axis. Considering only the 2D case (the following result can be extended to 3D), Equation (7) can be expressed by two scalar equations:

$$
\ddot{x}=v_{y}\left(\frac{d v_{y}}{d y} \cdot \frac{d}{d y}+v_{y} \frac{d^{2}}{d y^{2}}\right) P(y)
$$

and,

$$
\begin{aligned}
\ddot{y} & =v_{y}\left(\frac{d v_{y}}{d y} \cdot \frac{d y}{d y}+v_{y} \frac{d^{2} y}{d y^{2}}\right) \\
& =v_{y} \frac{d v_{y}}{d y}
\end{aligned}
$$


Focusing on Equation (8), if the vertical acceleration $a_{x}$ and horizontal velocity $v_{y}$ as a function of $y$, can be estimated, then Equation (8) can be solved for the path $P(y)$ by defining $s \equiv d P / d y=d x / d y$ :

$$
\begin{aligned}
\frac{a_{x}}{v_{y}} & =\left(\frac{d v_{y}}{d y} \cdot \frac{d}{d y}+v_{y} \frac{d^{2}}{d y^{2}}\right) P(y) \\
& =s \frac{d v_{y}}{d y}+v_{y} \frac{d s}{d y} \\
& =\frac{d}{d y}\left(v_{y} s\right)
\end{aligned}
$$

Equation (10) can be integrated once to solve for $s(y)$ :

$$
v_{y} S=\int_{y_{0}}^{y} \frac{a_{x}\left(y^{\prime}\right)}{v_{y}\left(y^{\prime}\right)} d y^{\prime}+C_{1}
$$

Integrating once again yields the trajectory path function with two free boundary value constants, $C_{1}$ and $C_{2}$ :

$$
\begin{gathered}
s(y)=\frac{d P(y)}{d y} \\
=\frac{1}{v_{y}(y)} \int_{y_{0}}^{y} \frac{a_{x}\left(y^{\prime}\right)}{v_{y}\left(y^{\prime}\right)} d y^{\prime}+C_{1} \\
P(y)=\int_{y_{0}}^{y}\left(\frac{1}{v_{y}\left(y^{\prime \prime}\right)} \int_{y_{0}}^{y^{\prime \prime}} \frac{a_{x}\left(y^{\prime}\right)}{v_{y}\left(y^{\prime}\right)} d y^{\prime}\right) d y^{\prime \prime}+C_{1} y+C_{2}
\end{gathered}
$$

The constants of integration can be found by specifying the initial starting point and the slope of the initial starting point. Specify the slope and solve for $C_{1}$ :

$$
s\left(y_{0}\right)=s_{0}
$$

Then solve for $C_{2}$ by specifying the initial starting value:

$$
P\left(y_{0}\right)=x_{0}
$$

\section{Application to CFD (Fluent $\left.{ }^{T M}\right)$ Based Trajectories}

If the vertical acceleration $a_{x}$ as a function of horizontal distance $y$ is approximated as a power law of the form:

$$
a_{x}(y) \approx \alpha y^{-\beta}-g \quad,
$$

where $g$ is gravity, and while approximating the horizontal velocity $v_{y}$ as a constant $v_{0}$, the double integral of Equation (13) can be evaluated. If the power law exponent $\beta$ is set equal to 2 , then a further simplified form of Equation (12) results: 


$$
\begin{aligned}
s(y) & =\frac{1}{v_{y}(y)} \int_{y_{0}}^{y} \frac{a_{x}\left(y^{\prime}\right)}{v_{y}\left(y^{\prime}\right)} d y^{\prime}+C_{1} \\
& =C_{1}-\left(\frac{\alpha}{y}+g y\right) / v_{0}^{2}
\end{aligned}
$$

Applying the boundary condition of Equation (14) results in

$$
C_{1}=s_{0}+\left(g y_{0}+\alpha / y_{0}\right) / v_{0}^{2} \quad .
$$

Finally, integrating Equation (13) gives the path $P(y)$ :

$$
P(y)=C_{1} y+C_{2}-\left(\frac{g y^{2}}{2}+\alpha \ln y\right) / v_{0}^{2} .
$$

Applying the boundary condition of Equation (15) results in the following:

$$
\begin{aligned}
C_{2} & =x_{0}+\left(\frac{g y_{0}^{2}}{2}+\alpha \ln y_{0}\right) / v_{0}^{2}-C_{1} y_{0} \\
& =x_{0}-s_{0} y_{0}+\left(\alpha\left(\ln y_{0}-1\right)-\frac{g y_{0}^{2}}{2}\right) / v_{0}^{2}
\end{aligned}
$$

Combining Equations (18), (19), and (20) gives a complete trajectory path equation:

$$
\begin{aligned}
P(y) & =\left(s_{0}+\left(g y_{0}+\alpha / y_{0}\right) / v_{0}^{2}\right) y+x_{0}-s_{0} y_{0}+\left(\alpha\left(\ln y_{0}-1\right)-\frac{g y_{0}^{2}}{2}\right) / v_{0}^{2}-\left(\frac{g y^{2}}{2}+\alpha \ln y\right) / v_{0}^{2} \\
& =x_{0}+s_{0}\left(y-y_{0}\right)+\frac{\alpha}{v_{0}^{2}}\left(\frac{y-y_{0}}{y_{0}}-\ln \frac{y}{y_{0}}\right)-\frac{g\left(y-y_{0}\right)^{2}}{2 v_{0}^{2}}
\end{aligned}
$$

Comparison of Equation (21) with the Equation (A-1) describing a previous empirical model shows that most parameters are with the exception of $\alpha$ in this model and $b$ in the empirical model. In the empirical model, the quantity $b x_{0} / y_{0}$ describes the tangent of the final trajectory angle in the limiting case of $g=0$, which is approximately the case for large horizontal velocities within the CFD simulation domain:

$$
\theta=\tan ^{-1} \frac{b x_{0}}{y_{0}}
$$

The parameter $\alpha$ in Equation (21) can be expressed in terms of $b$ by taking the limit of the derivative of the path function as $y$ goes to infinity with $g=0$ :

$$
\left.\frac{b x_{0}}{y_{0}} \equiv \lim _{y \rightarrow \infty} \frac{d}{d y} P(y)\right|_{g=0}=s_{0}+\frac{\alpha}{y_{0} v_{0}^{2}} \quad .
$$

Solving for $\alpha$ and substituting into Equation (21) results in a trajectory path formula similar to the previous empirical formula, but now based on a true physical model: 


$$
x(y)=x_{0}+s_{0}\left(y-y_{0}\right)+\left(b x_{0}-s_{0} y_{0}\right)\left(\frac{y-y_{0}}{y_{0}}-\ln \frac{y}{y_{0}}\right)-\frac{g\left(y-y_{0}\right)^{2}}{2 v_{0}^{2}} .
$$

\section{Results and Discussion}

The model described by Equation (24) is based on the assumption that the vertical acceleration $a_{x}$ as a function of horizontal distance $y$ is approximated by a power law as described by Equation (16), along with a horizontal velocity $v_{y}$ that is described by a constant $v_{0}$. For a practical application of this model, it is not necessary to find a fit to the vertical acceleration since the method previously used in the empirical model fitting can again be applied to this case. In this way, the particle trajectory path is determined by two primary fitting parameters, $s_{0}$ and $b$. For very small horizontal velocities, $v_{0}$ may also become a fitting parameter.
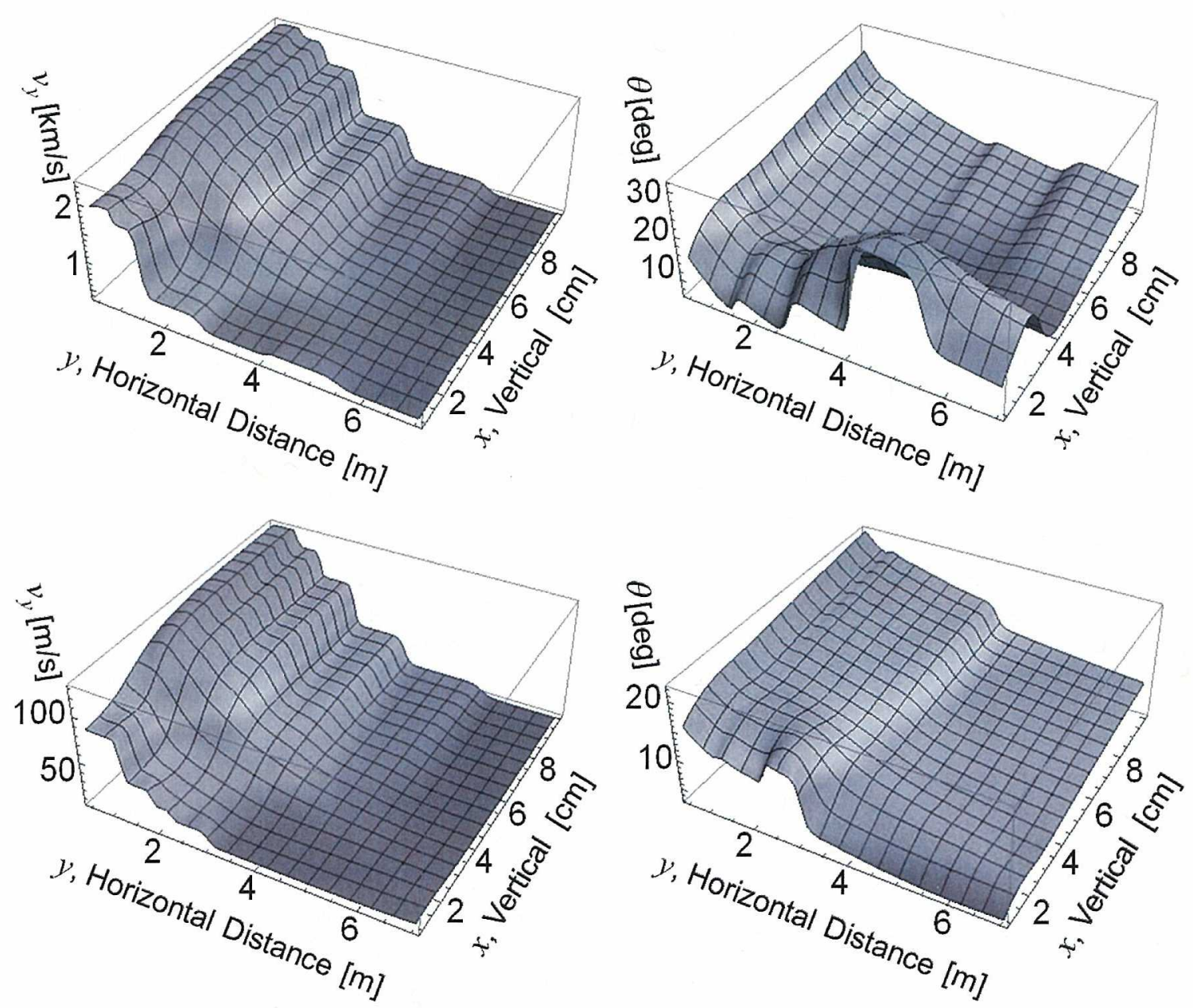

Figure 3. Application of the parameter matrix interpolation of Equation (4) using the Imperical Trajectory Model of Equation (1), operating on the output of a Fluent CFD simulation of rocket with a nozzle height of $5 \mathrm{ft}$ and a thrust of $67 \mathrm{~N}$. Top: particle $D=1 \mu \mathrm{m}$; bottom: particle $D=1000 \mu \mathrm{m}$. 

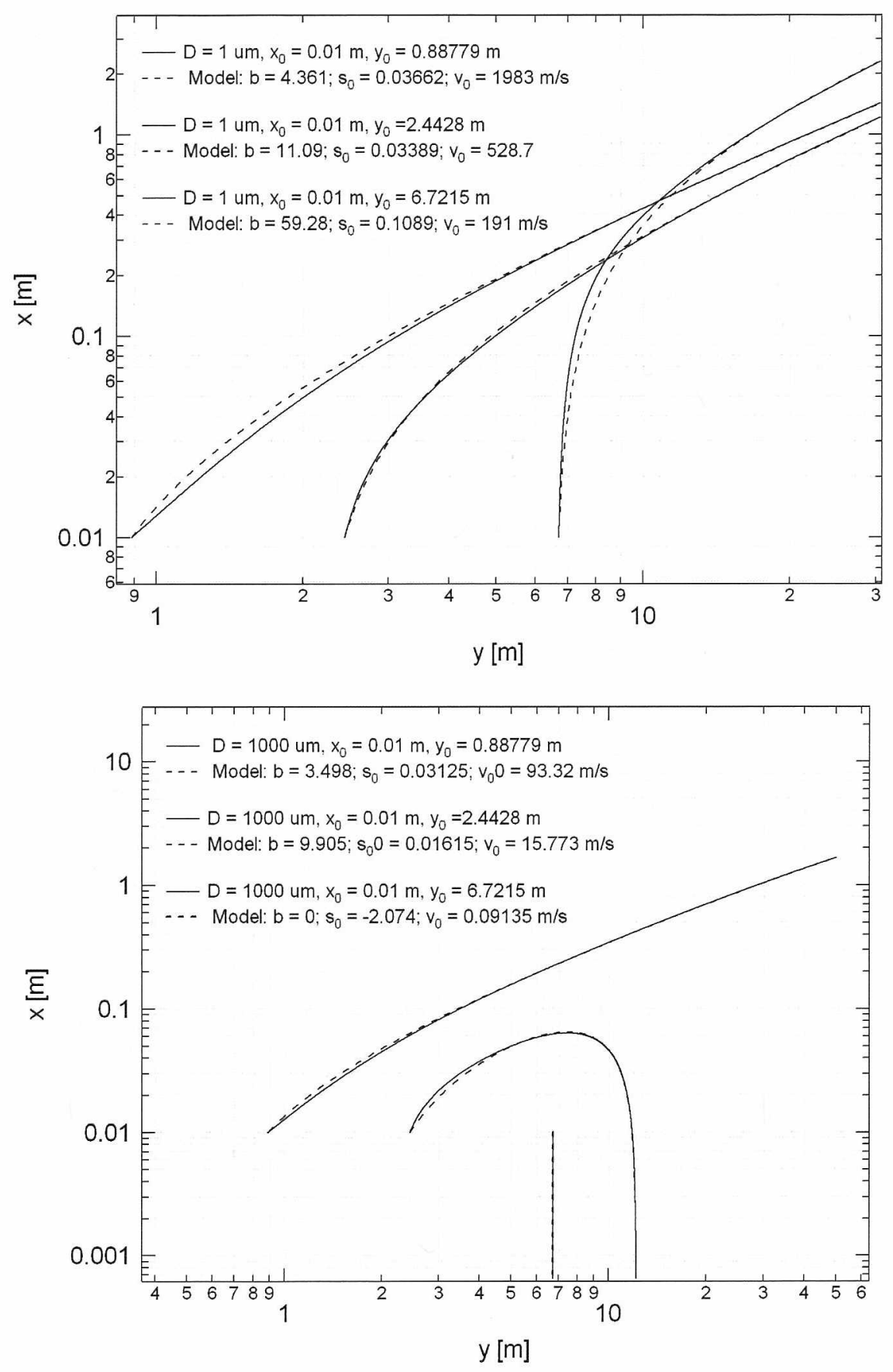

Figure 4. Solid lines: Full particle trajectories generated from post-processing of CFD simulations; dashed lines: trajectories generated by fitting the model of Equation (24) to solid lines. Top: $D=1 \mu \mathrm{m}$ and $x_{0}=$ $0.01 \mathrm{~m}$; bottom: $D=1000 \mu \mathrm{m}$ and $x_{0}=0.01 \mathrm{~m}$.

With the example parameter interpolation matrix of Table 1, Equations (4) and (5) define a procedure to estimate the trajectory path of particles using either the empirical trajectory model of Equation (1) or the physical trajectory model of Equation (24). The number and extent of model variables is determined by the extent of the parameter interpolation matrix. In this example, only three particle variables were considered for simplicity of description, $D, x_{0}$, and $y_{0}$. Figure 3 shows the application of the parameter 
matrix interpolation of Equation (4) using the ETP model of Equation (1), operating on the output of a Fluent CFD simulation of a rocket with a nozzle height of $5 \mathrm{ft}$ and a thrust of $67 \mathrm{~N}$. The top set of plots corresponds to a particle of $D=1 \mu \mathrm{m}$, while the bottom plot is for a $D=1000 \mu \mathrm{m}$ particle. The left plot is the parameter $v_{y}$, corresponding to a constant horizontal velocity. The right plot is the gravity free trajectory angle, defined by Equation (2). The stair-stepped appearance of the plot is a consequnece of the coarse matrix used (Table 1) with $N=21$. A larger matrix would smooth out the plot. Also, the simple Shepard's interpolation defined by Equation (4) is known to generate non-smooth surfaces since the gradient at all reference points (all point defined by $\mathbf{P}_{i}$ ) is equal to zero because of Equation (4). A quadratic variation of Shepard's interpolation (Renka, 1988) corrects this problem, but at the expense of a more complex algorithm.

\section{Conclusions}

In the process of verifying and characterizing the physical trajectory path model with Fluent test cases and comparing it to the previous empirical trajectory path model, the PTP model can be characterized by the following points:

- The PTP model has a physical basis, starting with simplified estimates of vertical acceleration and horizontal velocity. The input parameters are $x_{0}$ and $y_{0}$ (initial starting point); $s_{0}$ derivative of trajectory $d x(y) / d y$, evaluated at $y=y_{0}$; and $b$ where $b x_{0} / y_{0}$ is the final trajectory angle before gravity pulls the particle down. Note that $s_{0}$ and $b$ are the primary curve-fitting parameters. The final parameter is $v_{0}$, an assumed constant horizontal velocity and is a secondary curve fitting parameter.

- This model is time independent, showing vertical position $x$ as a function of horizontal distance $y$, by $x(y)=f(y)$, as described by Equation (24). The first term on the right is due to simple ballistics and a spherically expanding rarefied gas so that the trajectory is a straight line intersecting $(0,0,0)$, which is the point directly under the nozzle center on the surface. This term is generally the $<3^{\circ}$ trajectory angle. The second term on the right is due to vertical acceleration, which may be positive or negative. The last term on the right is the gravity term, which for a particle with velocities less than escape velocity will eventually bring the particle back to the ground.

- This model, even though it has the same number of parameters as the ETP model, seems to better describe the particle trajectories, even though it is far from perfect. Perfection in this context is traded for simplicity.

\section{References}

Lane, JE; Metzger, PT; and Carlson, JW; (2010), "Lunar Dust Particles Blown By Lander Engine Exhaust in Rarefied and Compressible Flow", 12th ASCE Aerospace Division International Conference, Honolulu, HI, March 14-17, 2010. Proceedings.

Loth, E. (2008_a), "Compressibility and Rarefaction Effects on Drag of a Spherical Particle”, AIAA Journal, 46(9), pp. 2219-2228.

Loth, E. (2008_b), "Lift of a Spherical Particle Subject to Vorticity and/or Spin", AIAA Journal, 46(4), pp. 801-809.

Renka, R. J (1988), "Multivariate Interpolation of Large Sets of Scattered Data", ACM Trans. Math. Software, 14(2), pp. 139-148.

Shepard, Donald (1968), "A Two-Dimensional Interpolation Function for Irregularly-Spaced Data", Proceedings of the 1968 23rd ACM national conference, Association for Computing Machinery, ACM Press, New York, NY, pp. 517-524. 
The public reporting burden for this collection of information is estimated to average 1 hour per response, including the time for reviewing instructions, searching existing data sources, gathering and maintaining the data needed, and completing and reviewing the collection of information. Send comments regarding this burden estimate or data sources, gathering and maintaining the data needed, and completing and reviewing the collection of information. Send comments regarding this burden estim

Directorate for Information Operations and Reports (0704-0188), 1215 Jefferson Davis Highway, Suite 1204, Arlington, VA 22202-4302. Respondents should be aware that notwithstanding any other provision of law, no person shall be subject to any penalty for failing to comply with a collection of information if it does not display a currently valid $O M B$ control number.

PLEASE DO NOT RETURN YOUR FORM TO THE ABOVE ADDRESS.
1. REPORT DATE (DD-MM-YYYY)
2. REPORT TYPE
23-07-2010
Publish
3. DATES COVERED (From - To)
July 2010

4. TITLE AND SUBTITLE

Ballistics Medel for Particles on a Horizontal Plane in a Vacuum Propelled by a

Vertically Impinging Gas Jet

5a. CONTRACT NUMBER

NAS10-03006

5b. GRANT NUMBER

5c. PROGRAM ELEMENT NUMBER

6. AUTHOR(S)

John E. Lane. ASRCA

Philip T. Metzger, NASA John F Kennedy Space Center

\section{5d. PROJECT NUMBER}

5e. TASK NUMBER

\section{5f. WORK UNIT NUMBER}

7. PERFORMING ORGANIZATION NAME(S) AND ADDRESS(ES)

ASRC Aerospace

Kennedy Space Center FL 32899

9. SPONSORING/MONITORING AGENCY NAME(S) AND ADDRESS(ES)

NASA John F Kennedy Space Center, FL 32899

10. SPONSORING/MONITOR'S ACRONYM(S)

11. SPONSORING/MONITORING REPORT NUMBER

\section{PERFORMING ORGANIZATION} REPORT NUMBER

\section{DISTRIBUTION/AVAILABILITY STATEMENT}

Public Domain

\section{SUPPLEMENTARY NOTES}

\section{ABSTRACT}

A simple trajectory model has been developed and is presented. The particle trajectory path is estimated by computing the vertical position as a function of the horizontal position using a constant horizontal velocity and a vertical acceleration approximated as a power law.

\section{SUBJECT TERMS}

lunar surface, regolith, ballistics, particle trajectory, equation of motion, rarfield gas jet

\begin{tabular}{|c|c|c|c|}
\hline \multicolumn{2}{|l|}{ 16. SECURITY CLASSIFICATION OF: } & 17. LIMITATION OF \\
ABSTRACT \\
a. REPORT & b. ABSTRACT & c. THIS PAGE & \\
$\mathrm{U}$ & $\mathrm{U}$ & $\mathrm{U}$ & $\mathrm{UU}$ \\
\hline
\end{tabular}

\begin{tabular}{l|l} 
18. NUMBER \\
OF \\
PAGES \\
\cline { 2 - 2 }
\end{tabular}

19b. NAME OF RESPONSIBLE PERSON Pasquale Ferrari 19b. TELEPHONE NUMBER (Include area code) 